Pesq. Vet. Bras. 37(11):1287-1291, novembro 2017 DOI: $10.1590 / \mathrm{S} 0100-736 X 2017001100015$

\title{
Avaliação clínica, endoscópica e histopatológica de cães com doença inflamatória intestinal ${ }^{1}$
}

\author{
Clarice M. Cascon², Marcela F.V. Mello ${ }^{3}$, Juliana S. Leite ${ }^{4}$ e Ana M.R. Ferreira ${ }^{2,5 *}$
}

\begin{abstract}
Cascon C.M., Mello M.F.V., Leite J.S. \& Ferreira A.M.R. 2017. [Clinical, endoscopic and histopathological evaluation of dogs with inflammatory bowel disease.] Avaliação clínica, endoscópica e histopatológica de cães com doença inflamatória intestinal. Pesquisa Veterinária Brasileira 37(11):1287-1291. Departamento de Patologia e Clínica Veterinária, Faculdade de Veterinária, Universidade Federal Fluminense, Rua Vital Brazil 64, Niterói, RJ 24230-340, Brazil. E-mail: anatopatovet@vm.uff.br

The aim of this study was to evaluate macro and microscopic gastrointestinal mucosa of domiciled dogs, with chronic gastrointestinal symptoms by endoscopy and histopathological evaluation, correlating clinical signs, macro and microscopy characteristics/aspects/ changes of duodenal and gastric mucosa. Twenty dogs of different breeds were evaluated with with chronic characteristic signs of Inflammatory Bowel Disease (IBD), being 7 males and 13 females, with ages ranging from 1.7 to 15.8 years old. The study was prospective and cross-sectional, conducted in dogs treated at the Teaching Veterinary Medicine Hospital of the Universidade Federal Fluminense (HUVET-UFF) and private clinics in the city of Rio de Janeiro. Gastroduodenal mucosal samples were obtained by endoscopic biopsy and were processed by routine histological technique and stained with hematoxylin- eosin and Giemsa. The samples were submitted to histopathological analysis for the detection of spiral bacteria suggestive of Helicobacter spp. The rapid urease test was also performed. The clinical signs, the macro and microscopic findings were compared and correlated. In this study, we established a positive correlation between weight loss and the presence of erythema in the antrum, body and duodenum as well as correlation between change in appetite and the presence of gastric inflammatory infiltrate and positivity in the urease test. This study demonstrated the importance of the observation of some clinical signs such as weight loss and appetite changes in the course of IBD. Thus, it is emphasized that dogs showing these clinical signs should have the IBD included in the differential diagnoses group avoiding thereby neglecting a disease that increasingly affects small animals.
\end{abstract}

INDEX TERMS: Bowel disease, dogs, vomiting, diarrhea, endoscopy, Canis familiaris, inflammatory infiltrate.

\footnotetext{
${ }^{1}$ Recebido em 19 de outubro de 2015.

Aceito para publicação em 9 de dezembro de 2016.

${ }^{2}$ Programa de Pós-Graduação em Medicina Veterinária (Clínica e Reprodução Animal), Departamento de Patologia e Clínica Veterinária, Setor de Anatomia Patológica Veterinária, Faculdade de Veterinária, Universidade Federal Fluminense (UFF), Av. Alm. Ary Parreiras 507, Icaraí, Niterói, RJ 24220-000, Brasil.

${ }^{3}$ Professora Adjunta do Departamento de Patologia e Clínica Veterinária, Setor de Anatomia Patológica Veterinária, UFF, Rua Miguel de Frias 9, Icaraí, Niterói, RJ 24220-900, Brasil.

${ }^{4}$ Professora Titular do Departamento de Patologia e Clínica Veterinária, Setor de Anatomia Patológica Veterinária, UFF, Rua Miguel de Frias 9, Icaraí, Niterói, RJ 24220-900, Brasil.

${ }^{5}$ Setor de Anatomia Patológica Veterinária, Faculdade de Veterinária, Universidade Federal Fluminense. Rua Vital Brazil 64, Niterói, RJ 24230340, Brasil. *Autor para correspondência: anatopatovet@vm.uff.br
}

RESUMO.- 0 presente estudo teve como objetivo avaliar macro e microscopicamente a mucosa gastrintestinal de cães domiciliados, que apresentaram sinais gastrintestinais crônicos, por meio de exame endoscópico e da avaliação histopatológica. Os sinais clínicos, a macro e a microscopia das mucosas gástrica e duodenal foram correlacionados. Foram avaliados 20 cães de diferentes raças, sendo 7 machos e 13 fêmeas, com idade variando entre 1,7 a 15,8 anos, que apresentavam cronicidade dos sinais característicos da Doença Inflamatória Intestinal (DII). 0 estudo foi prospectivo e transversal, realizado em cães atendidos no Hospital Universitário de Medicina Veterinária da Universidade Federal Fluminense (HUVET - UFF) e em clínicas privadas do município do Rio de Janeiro. Amos- 
tras de mucosa gastroduodenal foram obtidas por meio de biópsia endoscópica e foram processadas por técnica histológica de rotina e coradas por Hematoxilina-Eosina e Giemsa. As amostras foram submetidas à análise histopatológica para pesquisa de bactérias espiraladas sugestivas de Helicobacter spp. 0 teste rápido da urease também foi realizado. Os sinais clínicos, e os achados macro e microscópicos foram confrontados e correlacionados. Neste estudo, foi possível estabelecer correlação positiva entre a perda de peso e a presença de enantema no antro, no corpo e no duodeno além de correlação entre alteração do apetite, a presença de infiltrado inflamatório gástrico e a positividade no teste da urease. Este estudo demonstrou a importância da observação de alguns sinais clínicos como a perda de peso e alterações do apetite no curso da Doença Inflamatória Intestinal. Desta forma, enfatiza-se que cães que apresentem tais sinais clínicos devem ter a Doença Inflamatória Intestinal incluída no grupo de diagnósticos diferenciais evitando, com isso, negligenciar uma enfermidade que, cada vez mais, acomete animais de pequeno porte.

TERMOS DE INDEXAÇÃO: Doença inflamatória intestinal, caninos, vômito, diarreia, endoscopia, Canis familiaris, Infiltrado inflamatório.

\section{INTRODUÇÃOO}

Doença Inflamatória Intestinal (DII) é um termo aplicado a distúrbios gastrointestinais idiopáticos, crônicos, caracterizados por inflamação da mucosa (Jergens et al. 1992, Jergens 1999). Na Medicina Veterinária, o conhecimento acerca de suas várias manifestações se expandiu nos últimos 30 anos. 0 reconhecimento de que esta síndrome é uma doença, altamente, prevalente em cães e gatos só ocorreu em meados de 1980.

A hipótese diagnóstica ocorre em animais que apresentam sinais clínicos crônicos tais como diarreia, vômito, perda de peso e alterações do apetite sendo confirmada pela presença de infiltrado inflamatório na lâmina própria na histopatologia, incluindo linfócitos, plasmócitos, eosinófilos, neutrófilos e macrófagos (Willard et al. 2008). 0 tipo de inflamação é determinado pela descrição microscópica com base nas células inflamatórias predominantemente envolvidas e na área do trato gastrintestinal afetada (Flesja \& Yri 1977). Para serem considerados crônicos, os sinais clínicos devem persistir por mais de 3 semanas (Washabau et al. 2010). Outras causas de gastroenterite devem ser excluídas por uma detalhada avaliação clínica e laboratorial. A alergia/intolerância alimentar também deve ser excluida através de terapia dietética com uma nova fonte de proteina ou proteina hidrolizada (Jergens 1999). A DII idiopática é a forma mais comum de doença intestinal crônica em cães (Willard et al. 2008).

Há algumas décadas, a endoscopia digestiva alta vem sendo utilizada nos Estados Unidos e Europa na clínica de pequenos animais como importante ferramenta diagnóstica para avaliação gastrintestinal e coleta de material para exame histopatológico, permitindo, de forma minimamente invasiva, o diagnóstico de inúmeras enfermidades (Tams 2005). No Brasil, a utilização do exame endoscópico vem crescendo nos últimos anos, mas seu uso ainda é limitado devido ao alto custo do aparelho, dificuldade na aprendizagem da técnica e infrequente solicitação do exame (Silva \& Machado 2015). A endoscopia, apesar de permitir a avaliação da mucosa e coleta de material, tem limitações devido ao tamanho reduzido da amostra e por permitir somente a coleta de material de forma superficial além da necessidade de anestesia geral para a sua realização (Tams 2005). 0 presente estudo tem grande importância por unir dados clínicos, endoscópicos e histopatológicos a fim de melhorar o diagnóstico da DII e consequentemente direcionar o tratamento e melhorar qualidade de vida dos cães e de seus proprietários.

O presente estudo teve como objetivo avaliar macro e microscopicamente a mucosa gastrintestinal de cães domiciliados, com sintomatologia gastrointestinal crônica por meio de exame endoscópico e da avaliação histopatológica, e correlacionar os sinais clínicos, a macro e microscopia das mucosas gástrica e duodenal.

\section{MATERIAL E MÉTODOS}

Desenho do estudo. 0 estudo foi aprovado pelo Comitê de ética no uso de animais - CEUA/UFF sob no. 44/2012 e o termo de consentimento livre e esclarecido foi obtido dos proprietários dos cães participantes do estudo.

Foi realizado um estudo prospectivo, transversal, em 20 cães com idades entre 1,7 e 15,8 anos, que apresentavam sinais gastrintestinais crônicos tais com vômito, diarreia do intestino delgado, alteração do apetite e perda de peso atendidos no Hospital Universitário de Medicina Veterinária da Universidade Federal Fluminense (HUVET-UFF), Niterói/RJ (UFF) e em clínicas privadas do município do Rio de Janeiro - RJ. Em todos os cães participantes do estudo foram excluídas outras causas de gastroenterite, por meio de avaliação clínica e laboratorial e também foi realizado o teste de exclusão para alergia alimentar.

Foram utilizados como critérios de exclusão: cães submetidos à cirurgia do aparelho digestivo, cães que estivessem fazendo uso ou que tivessem utilizado nos últimos 30 dias anti-inflamatórios e/ou antibióticos, doenças que impedissem a realização de anestesia geral e enfermidades que, secundariamente, pudessem causar lesões no estômago e/ou intestino.

Endoscopia. Para o exame endoscópico, os animais foram submetidos a jejum hídrico de quatro horas e alimentar de 12 horas. Para a indução anestésica foi utilizado Propofol $(4 \mathrm{mg} / \mathrm{kg})$ e para manutenção anestésica foi utilizado Isofluorano.

Para a realização do exame, os cães foram posicionados em decúbito lateral esquerdo utilizando um fibroendoscópio flexível de $110 \mathrm{~cm}$ de comprimento e 9,8 mm de diâmetro, modelo FG-100FP da marca Fujinon ${ }^{\circledR}$. Na realização da endoscopia digestiva alta, foi avaliado macroscopicamente a hipofaringe, até a primeira porção do duodeno. Em seguida, foi lentamente retirado analisando-se os seguintes segmentos: corpo gástrico, pregas gástricas, grande curvatura e antro. A ponta do aparelho foi, parcialmente, retrofletida para avaliação da incisura angularis e da pequena curvatura e foi, totalmente, retrofletido para avaliar a cárdia. 0 aparelho foi retirado avaliando o esôfago. Durante o exame foram coletadas amostras de biópsia do corpo gástrico, antro pilórico e do duodeno.

A avaliação macroscópica da mucosa gástrica e duodenal foi descritiva. As lesões destes órgãos foram classificadas de acordo com o Consenso do Colégio Americano de Medicina Interna Veterinária (Washabau et al. 2010). 
Teste da uréase. Um fragmento coletado de cada região foi colocado em microtubos próprios do teste comercial Uretest ${ }^{\circledR}$, Renylab (Belo Horizonte, Minas Gerais) para realização do teste da urease. 0 teste foi considerado positivo quando a coloração se alterou de amarelo para rosa em um período máximo de 24 horas, indicando a presença de bactérias produtoras de urease.

Histopatologia. As amostras obtidas endoscopicamente foram fixadas em formol tamponado a $10 \%$ e pH 7,2 e, posteriormente foi realizado o processamento histotécnico de rotina para confecção de cortes histológicos de $5 \mu \mathrm{m}$, que foram posteriormente corados com Hemotoxilina-Eosina (HE) e pelo Giemsa modificado. As amostras coradas por HE foram avaliadas por um único observador em um aumento de 400x de acordo com o Consenso do Colégio Americano de Medicina Interna Veterinária (Washabau et al. 2010).

A pesquisa de Helicobacter spp. foi realizada com coloração Giemsa modificado no aumento de 1000x com óleo de imersão. As amostras foram avaliadas quanto à presença de microorganismos espiralados morfologicamente compatíveis com Helicobacter spp. nas formas bacilar ou cocóide.

Cálculo estatístico. Os dados foram analisados com o auxílio do programa SPSS para Windows versão 13. Comparações entre variáveis categóricas dicotômicas foram realizadas pelo teste do Qui-quadrado e Fischer; e as variáveis categóricas ordinárias (mais de dois níveis) foram analisadas pelo teste de correlação de Spearman com nível de significância de 5\%.

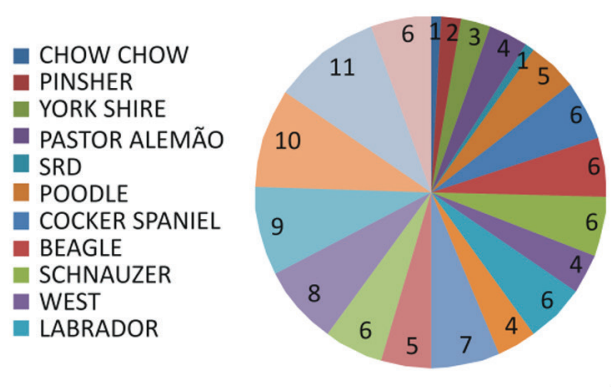

A

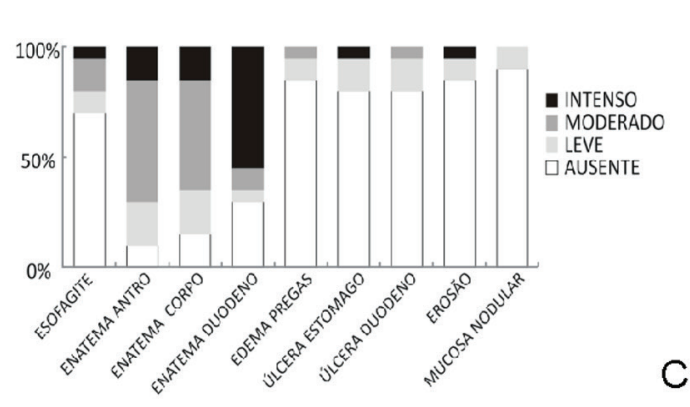

C

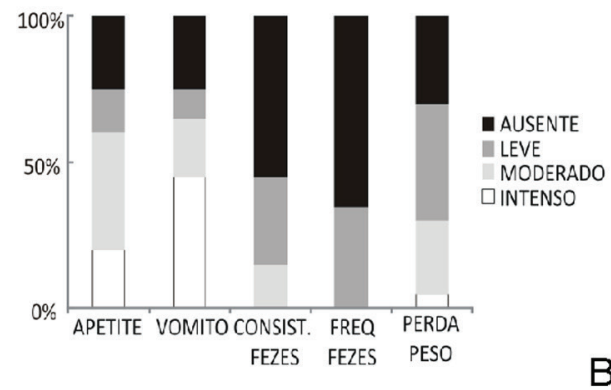

Foram estudados 20 cães sendo 13 fêmeas (65\%) e sete machos (35\%) com média de idade de $7 \pm 1$ ano $(1,7$ a 15,9 anos). A distribuição racial está demonstrada abaixo na Figura 1A.

Nove cães (45\%) apresentavam, simultaneamente, alteração na consistência das fezes e no número de evacuações. Destes, oito também tinham redução do apetite e perda de peso e cinco apresentavam vômitos. Os sinais clínicos estão representados na Figura 1B.

Em todos os animais, foram realizadas a avaliação endoscópica com a observação de possíveis lesões, e histopatológica da mucosa gastroduodenal. Os resultados da avaliação endoscópica da mucosa gastroduodenal estão representados na Figura 1C, e os resultados da avaliação histopatológica apresentados nas Figuras 1D e 1E.

Observou-se tendência à correlação das alterações na consistência das fezes dos cães com a presença de úlcera duodenal observada no exame endoscópico $(\mathrm{P}=0,057)$. A perda de peso foi correlacionada positivamente $(\mathrm{p}<0,05)$ com enantema de antro, enantema de corpo e com enantema de duodeno.

Quando correlacionados, os dados da avaliação histo-

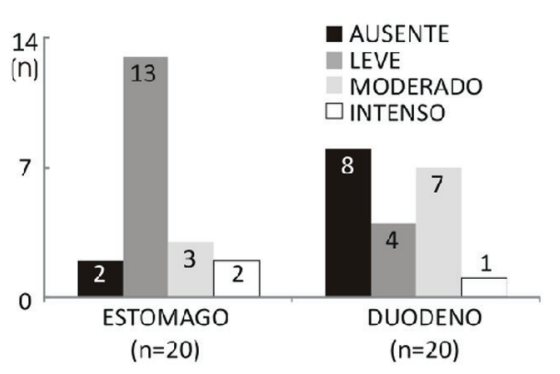

E

Fig.1. Cães com Doença Inflamatória Intestinal (n=20). (A) Percentual de distribuição racial, (B) Distribuição dos sinais clínicos, (C) Distribuição das alterações endoscópicas, (D) Distribuição das alterações histopatológicas, teste da urease (positivo) e presença de microorganismos espiralados sugestivos de Helicobacter spp., (E) Distribuição do infiltrado inflamatório linfocítico plasmocitário. 
patológica com as observações endoscópicas, houve correlação positiva $(\mathrm{p}<0,05)$ entre a presença de infiltrado inflamatório linfoplasmocitário gástrico e edema de pregas gástricas, erosão plana, enantema de corpo e a presença de líquido de estase. 0 infiltrado inflamatório linfoplasmocitário duodenal foi correlacionado positivamente com úlcera duodenal, com enantema de duodeno e com a presença de líquido de estase.

Confrontando os dados clínicos com a avaliação histopatológica houve correlação positiva $(\mathrm{p}<0,05)$ entre alteração do apetite e a presença de infiltrado inflamatório no estômago e entre edema de duodeno e positividade no teste da urease.

A maioria das raças presentes neste estudo foi descrita em trabalhos anteriores. Craven et al. (2004) estudaram 80 cães com evidências de infiltrado inflamatório gástrico, duodenal ou colônico, e as raças mais comumente afetadas foram: Pastor Alemão, Golden Retriever, West Highland White Terrier, Boxer, Labrador, Border Collie e Weimaraner. Já foi comprovada a predisposição genética à DII de várias raças caninas, tais como Pastores Alemães, Basenjis e Shar Peis (Jergens et al. 1992, Jergens 1999, German et al. 2000). Das raças encontradas em estudos prévios em cães com DII, algumas foram encontradas no presente trabalho como Pastor Alemão, West Highland White Terrier e Labrador.destacando a alta frequência dessa síndrome em cães de raça pura.

A raça Poodle não é descrita, em outros trabalhos, como pré-disposta à DII, porém no presente estudo foi a mais freqüente (30\%). Na análise deste fator, deve-se levar em consideração que, atualmente, no Brasil, é uma raça bastante difundida e estes convivem com grande proximidade com os proprietários permitindo, desta forma, que os mesmos observem quaisquer sinais de desordem gastrointestinal. Os animais que convivem dentro das residências, de uma forma geral, recebem mais atenção e cuidados dos proprietários, diferente de cães, como o Pastor Alemão, que vivem na área externa das residências brasileiras.

A média de idade dos cães estudados está de acordo com o descrito por outros autores como (Schreiner et al. 2008) que tiveram uma média de 6,9 anos. Jergens et al. (1992) encontraram média de idade de 6,3 anos e German et al. (2000) média de 6 anos de idade. Contudo, alguns cães no presente estudo tinham menos de 2 anos de idade o que sugere que a DII deve ser incluída como diagnóstico diferencial nas gastroenterites de cães jovens.

Vômito, diarreia e alterações de apetite são queixas comuns na rotina de atendimento de pequenos animais. São sinais clínicos inespecíficos que exigem exames clínicos e laboratoriais detalhados para o diagnóstico (Dye et al. 2013). Os achados são compatíveis com estudos anteriores como os de Jergens et al. (1992), e Jergens (1999) que descrevem como sinais clínicos mais comuns associados com doença crônica do intestino delgado, diarreia e perda de peso. No estudo realizado por Jergens (1999), Jergens et al. (1992) em que foram avaliados 58 cães com DII, 20 cães (34\%) apresentavam vômito, 16 (28\%) diarreia e 11 (19\%) tiveram perda de peso. Craven et al. (2004) classificaram 80 casos de acordo com a localização do infiltrado inflamatório, em sinais altos (gastrite e enterite), baixos (colite) e difusos (altos e baixos). Em todas as classificações os animais apresentaram múltiplos sinais clínicos, principalmente vômito e diarreia, associados frequentemente à perda de peso e alterações do apetite. Embora sejam sinais comuns, não há estudo de prevalência. 0 presente estudo não teve o objetivo de avaliar a prevalência dos sinais clínicos, porém o percentual encontrado demonstrou uma alta ocorrência dos mesmos.

Enantema, vascularização, edema, friabilidade, hemorragia, úlceras, erosões, conteúdo (muco/bile/alimento) e a passagem do aparelho pelo esfíncter gastroesofágico e pelo piloro foram parâmetros considerados critérios de avaliação endoscópica das mucosas gástrica e duodenal. Tais parâmetros foram descritos no Consenso do Colégio Americano de Medicina Interna Veterinária desenvolvido por Washabau et al. (2010). No presente estudo, 90\% dos cães apresentaram algum tipo de alteração endoscópica. 0 alto percentual encontrado de bactérias espiraladas no exame histopatológico com a coloração de Giemsa e de positividade no teste da urease sugere que Helicobacter spp. possa ser uma importante causa de gastrite em cães no Brasil, um país em desenvolvimento com alta prevalência da bactéria na população.

Willard et al. (2002) relataram que a avaliação histopatológica é subjetiva e que mesmo quando critérios específicos são aplicados, pode ocorrer grande variação de interpretação na avaliação das amostras de tecido gastrintestinal entre patologistas. Observaram falta de uniformidade na avaliação em mais de 50\% das amostras de biópsia quando examinadas por diferentes patologistas veterinários. Esta variação interpretativa cria problemas para o diagnóstico da doença gastrointestinal e também na monitoração do progresso terapêutico.

Washabau et al. (2010) descreveram que, para minimizar a subjetividade, algumas questões fundamentais no processo de diagnóstico são essenciais. A citar: o tamanho e a qualidade das amostas são adequados para um preciso diagnóstico?; qual é a origem da resposta inflamatoria (neutrofílica, eosinofílica, granulomatosa, piogranulomatosa ou linfoplasmocitária)?; quão grave é a resposta inflamatória?; e, quando uma resposta inflamatória pode ser a precursora de neoplasia linfóide? Desta forma, neste trabalho, em cada amostra o tamanho e a qualidade das amostas foram cuidadosamente selecionados, a origem da resposta inflamatória foi descrita, assim como a intensidade da resposta.

Quando comparados os sinais clínicos com as alterações endoscópicas observou-se no presente estudo que o sinal clínico mais comum no cão com gastrite é a perda de peso. Este é um dado importante para os clínicos de pequenos animais que geralmente observam este sinal tardiamente, quando o mesmo já produz grande alteração no escore corporal do animal.

Em humanos, a bactéria Helicobacter spp. é reconhecidamente a principal causa de úlcera gástrica (Peterson 1991). Todos os animais do presente estudo que apresentavam nodularidade de mucosa foram positivos no teste da urease. Este é um achado que já foi descrito em humanos 
como sugestivo de infecção por Helicobacter spp. (Peterson 1991). A alta prevalência de positividade nos testes da urease era esperada já que a incidência desta bactéria em cães é comprovadamente alta (Geyer et al. 1993, Hermanns et al. 1995, Hwang et al. 2002, Polanco et al. 2011). Jakobsson et al. (2007) relataram que a hiperplasia de folículo linfóide está associada à infecção por Helicobacter spp. Esta correlação sugere que as erosões observadas durante o exame endoscópico também possam ser em decorrência da infecção por esta bactéria.

Na correlação estabelecida neste estudo entre infiltrado inflamatório linfoplasmocitário duodenal com enantema de duodeno e úlcera duodenal foi possível perceber que as alterações histopatológicas geram secundariamente lesões macroscópicas que podem ser visualizadas na endoscopia e podem, após o exame endoscópico, aumentar a suspeita da DII. 0 mesmo pode ser sugerido quando o infiltrado inflamatório gástrico está presente e correlacionado com edema de pregas gástricas, erosão plana e enantema de corpo. Os infiltrados inflamatórios gástricos e duodenais foram correlacionados neste estudo com a presença de líquido de estase demonstrando que o infiltrado inflamatório também interfere na motilidade gastroduodenal. Essas correlações validam a hipótese de que as lesões macroscópicas observadas na endoscopia estão intimamente relacionadas e reforçam o diagnóstico da DII.

\section{CONCLUSÃO}

Cães adultos são mais acometidos pela Doença Inflamatória Intestinal, sendo a raça Poodle a de maior incidência no presente estudo, caracterizando, para esta raça, uma maior predisposição. Existe correlação entre os sinais clínicos, os achados endoscópicos e histopatológicos demonstrando a importância de uma correta avaliação clínica e da mucosa gastrointestinal para o diagnóstico definitivo dessa enfermidade em cães.

\section{REFERÊNCIAS}

Craven M., Simpson J.W., Ridyard A.E. \& Chandler M.L. 2004. Canine inflammatory bowel disease: retrospective analysis of diagnosis and outcome in 80 cases (1995-2002). J. Small Anim. Pract. 45(7):336-342.

Dye T.L., Diehl K.J., Wheeler S.L. \& Westfall D.S. 2013. Randomized, controlled trial of budesonide and prednisone for the treatment of idiopathic inflammatory bowel disease in dogs. J. Vet. Int. Med. 27 (6):13851391.
Flesja K. \& Yri T. 1977. Protein-losing enteropathy in the Lundehund. J. Small Anim. Pract. 18(1):11-23.

German A.J., Hall E.J. \& Day M.J. 2000. Relative deficiency in IgA production by duodenal explants from German shepherd dogs with small intestinal disease. Vet. Immunol. Immunopathol. 76(1/2):25-43.

Geyer C., Colbatzky F, Lechner J. \& Hermanns W. 1993. Occurrence of spiral-shaped bacteria in gastric biopsies of dogs and cats. Vet. Rec. 133(1):18-19.

Hermanns W., Kregel K., Breuer W. \& Lechner J. 1995. Helicobacter-like organisms: histopathological examination of gastric biopsies from dogs and cats. J. Comp. Pathol. 112(3):307-318.

Hwang C.Y., Han H.R. \& Youn H.Y. 2002. Prevalence and clinical characterization of gastric Helicobacter species infection of dogs and cats in Korea. J. Vet. Sci. 3(2):123-133.

Jakobsson H., Wreiber K., Fall K., Fjelstad B., Nyren O. \& Engstrand L. 2007. Macrolide resistance in the normal microbiota after Helicobacter pylori treatment. Scand. J Infect. Dis. 39(9):757-763.

Jergens A.E. 1999. Inflammatory bowel disease: current perspectives. Vet. Clin. North Am., Small Anim. Pract. 29(2):501-521, vii.

Jergens A.E., Moore F.M., Haynes J.S. \& Miles K.G. 1992. Idiopathic inflammatory bowel disease in dogs and cats: 84 cases (1987-1990). J. Am. Vet. Med. Assoc. 201(10):1603-1608.

Peterson W.L. 1991. Helicobacter pylori and Peptic Ulcer Disease. N. Engl. J. Med. 324:1043-1048.

Polanco R., Salazar V., Reyes N., García-Amado M.A. \& Michelangeli F. 2011. High prevalence of DNA from non-H. pylori helicobacter in the gastric mucosa of venezuelan pet dogs and its histological alterations. Revta Inst. Med. Trop. São Paulo 53:207-212.

Schreiner N.M., Gaschen F.G., Grone A., Sauter S.N. \& Allenspach K. 2008. Clinical signs, histology, and CD3-positive cells before and after treatment of dogs with chronic enteropathies. J. Vet. Intern. Med. 22(5):10791083.

Silva L.C. \& Machado V.M.V. 2015. O uso da endoscopia digestiva alta em pequenos animais. Vet. Zootec. 22(1):8-14.

Tams T.R. 2005. Endoscopia e Laparoscopia em Gastroenterologia Veterinária, p.93-113. In: Tams T.R. (Ed.), Gastroenterologia de Pequenos Animais. $2^{\underline{a}}$ ed. Roca, São Paulo.

Washabau R.J., Day M.J., Willard M.D., Hall E.J., Jergens A.E., Mansell J., Minami T., Bilzer T.W. \& W.I.G.S. Group 2010. Endoscopic, biopsy, and histopathologic guidelines for the evaluation of gastrointestinal inflammation in companion animals. J. Vet. Intern. Med. 24(1):10-26.

Willard M.D., Jergens A.E., Duncan R.B., Leib M.S., McCracken M.D., DeNovo R.C., Helman R.G., Slater M.R. \& Harbison J.L. 2002. Interobserver variation among histopathologic evaluations of intestinal tissues from dogs and cats. J Am. Vet. Med. Assoc. 220(8):1177-1182.

Willard M.D., Mansell J.M., Fosgate G.T., Gualtieri M., Olivero D., Lecoindre P., Twedt D.C., Collett M.G., Day M.J., Hall E.J., Jergens A.E., Simpson J.W., Else R.W. \& Washabau R.J. 2008. Effect of sample quality on the sensitivity of endoscopic biopsy for detecting gastric and duodenal lesions in dogs and cats. J. Vet. Intern. Med. 22(5):1084-1089. 